\title{
La importancia de la actuación médico-legal en la donación y trasplante de órganos y tejidos
}

\section{Alonso Gil}

Coordinador Autonómico de Trasplantes de Andalucía. Servicio Andaluz de Salud.

Correspondencia:

Manuel Alonso Gil

E-mail: manuel.alonso.sspa@ juntadeandalucia.es
El trasplante de órganos y tejidos constituye hoy día una actividad terapéutica perfectamente consolidada, al menos en los países desarrollados. El único factor limitante para satisfacer la importante demanda del número de trasplantes, que crece de manera exponencial a medida que mejoran los resultados de supervivencia, es la escasez de órganos y tejidos.

La donación-trasplante constituye un clásico prototipo de lo que significa el trabajo en equipo. El desarrollo del proceso comienza con la detección del potencial donante, pasa por las distintas fases del mismo y termina con el trasplante de los distintos órganos y tejidos (Figura 1). Resulta obvio manifestar que todas las actividades tienen que estar perfectamente coordinadas y sincronizadas, y cada profesional o grupo implicado tiene que desarrollar su trabajo de forma precisa y en un corto periodo de tiempo. Son más de 100 profesionales sanitarios y no sanitarios los que participan en cada uno de estos procesos. La interrupción en cualquiera de sus fases implicará la pérdida final del donante, que es decir lo mismo que dejar de salvar varias vidas humanas.

Hay que significar que España es, de manera ininterrumpida, líder mundial en donación de órganos y tejidos desde hace 23 años. En lo que respecta a Andalucía, a pesar de empezar la era de los trasplantes 14 años más tarde que en Madrid o Cataluña, lo que significó un considerable retraso en el desarrollo de esta compleja actividad asistencial, tiene ya una tasa de donación de órganos superior a la media española en los últimos 5 años de manera consecutiva. Los resultados tanto de España como de Andalucía determinan que nuestras tasas de donación sean el doble que la media de los países de la Unión Europea, por lo que tenemos el doble de posibilidades de un trasplante en el caso de requerirlo.

Y no es fruto del azar que seamos líderes mundiales de manera continuada en el transcurso de los años en una actividad tan compleja. Nada es gratis, y todo requiere un esfuerzo y un trabajo. No obstante, además de lo anterior, es preciso significar que el éxito de lo que se ha venido en llamar en el contexto internacional "el modelo español" obedece a una serie de singularidades, siendo las más importantes las siguientes:

- Una legislación, que empieza con la Ley 30/1979, de 27 de octubre, sobre Extracción y Trasplante de Órganos, que resulta adecuada y es similar a la de otros países occidentales, con una clara definición de la muerte encefálica y en parada cardiorrespiratoria, de las condiciones de extracción de órganos, de la ausencia de motivación económica, etc.

- Una red de coordinadores de trasplante a tres niveles: nacional, autonómico y hospitalario, siendo quizás la figura de este último una de nuestras señas de identidad. En su inmensa mayoría son especialistas en cuidados intensivos y tienen una dedicación laboral parcial en las tareas de coordinación.

- Los grandes esfuerzos encaminados a la formación continuada, tanto de los coordinadores como de gran parte del personal sanitario y no sanitario, con cursos generales y específicos de cada uno de los pasos del proceso: detección de donantes, aspectos legales, entrevista familiar, aspectos organizativos, gestión, comunicación...

- Un exquisito trato con los medios de comunicación con el fin de mejorar el conocimiento de la población española sobre la donación y el trasplante.

- Disponer de un Sistema Nacional de Salud de cobertura universal.

Como hemos dicho con anterioridad, estos excelentes resultados son debidos a la formidable labor de los distintos profesionales y colectivos que intervienen en el proceso de donación-trasplante. $Y$ en este sentido es necesario destacar el impecable trabajo 


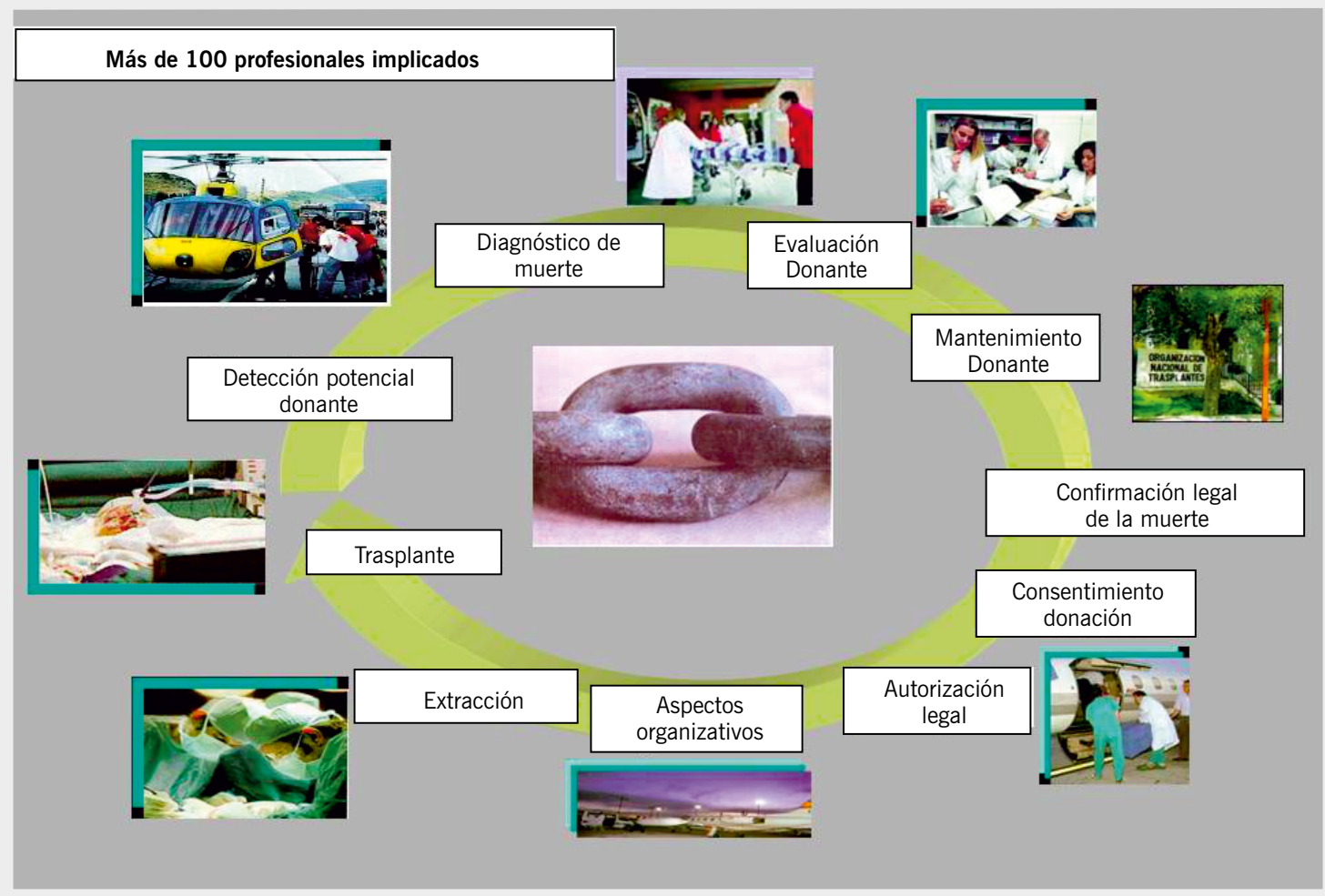

Figura 1.

El proceso de donación y trasplante. que desarrollan jueces y forenses. La colaboración que siempre han prestado tanto a la Organización Nacional de Trasplantes como a las distintas coordinaciones autonómicas y hospitalarias de trasplantes ha sido extraordinaria y muy positiva. Y lo digo no sólo por mi propia experiencia como Coordinador Sectorial de Trasplantes en Cádiz en la década de 1990 y después y en la actualidad como Coordinador Autonómico de Trasplantes de Andalucía, sino también por el reducido número de negativas judiciales que se dan cada año tanto en Andalucía como en España. En efecto, en Andalucía, en los últimos 10 años se han producido 11 negativas judiciales (Tabla 1 ), con una media de una negativa por año, que representan sólo un $1,8 \%$ de las 624 autorizaciones solicitadas. Aclarar que sólo el $23 \%$ de los donantes de órganos son casos judiciales, que requieren por lo tanto el preceptivo informe del médico forense y la correspondiente autorización del juez. Las estadísticas de Andalucía son similares a las de España. Así, en el pasado año 2014, se produjeron en España seis negativas judiciales (de ellas, cuatro en Cataluña), lo que implica un porcentaje del $1,7 \%$ de las 363 autorizaciones solicitadas (Figura 2).
Es más que probable que el escaso número de negativas judiciales se deba, al menos en parte, al excelente clima de colaboración que existe desde siempre entre jueces, forenses y coordinadores de trasplantes. Son ya sobradamente conocidas las Jornadas de encuentro entre profesionales de la Administración de Justicia y de la comunidad trasplantadora, que se llevan a cabo de forma anual en Santander desde 1997. También en Andalucía hemos organizado jornadas de trabajo, casi siempre coincidiendo con la publicación de nuevos decretos y con la finalidad de una mejor puesta en común del trabajo de los diferentes colectivos implicados. $Y$ hemos detectado con enorme satisfacción la magnífica acogida de estos cursos o jornadas, donde los ponentes son jueces, forenses y coordinadores, y los oyentes profesionales de los tres colectivos citados. De hecho, el éxito obtenido en una reunión con carácter autonómico que se llevó a cabo el 27 y 28 de noviembre del pasado año en Córdoba, donde acudió una representación de los profesionales implicados de cada provincia, propició que se solicitara la realización de estas reuniones con carácter provincial para que el contenido de las mismas pudiera llegar a todos los profesionales que así lo desearan. De esta forma, ya en el presente año 
Tabla 1. Datos negativas judiciales. Andalucía 01-01-2006 a 31-05-2015. Donantes en muerte encefálica + donantes en asistolia.

\begin{tabular}{|c|c|c|c|c|c|}
\hline & $\begin{array}{c}\text { Casos } \\
\text { judiciales }\end{array}$ & $\begin{array}{l}\text { Negativas } \\
\text { judiciales }\end{array}$ & $\begin{array}{c}\% \text { Negativas } \\
\text { judiciales sobre } \\
\text { el total de casos } \\
\text { judiciales }\end{array}$ & $\begin{array}{c}\text { Total } \\
\text { donantes }\end{array}$ & $\begin{array}{c}\% \text { casos } \\
\text { judiciales sobre } \\
\text { el total de } \\
\text { donantes }\end{array}$ \\
\hline 2006 & 84 & $1(\mathrm{MA})$ & 1,2 & 261 & 32,2 \\
\hline 2007 & 58 & 1 (SE) & 1,7 & 247 & 23,5 \\
\hline 2008 & 59 & $1(\mathrm{MA})$ & 1,7 & 282 & 20,9 \\
\hline 2009 & 72 & $3(\mathrm{MA}, \mathrm{SE}, \mathrm{CA})$ & 4,2 & 295 & 24,4 \\
\hline 2010 & 56 & 0 & 0,0 & 264 & 21,2 \\
\hline 2011 & 59 & 0 & 0,0 & 311 & 19,0 \\
\hline 2012 & 72 & 0 & 0,0 & 307 & 23,5 \\
\hline 2013 & 63 & 4 (3 AL, GR) & 6,3 & 297 & 21,2 \\
\hline 2014 & 66 & 0 & 0,0 & 315 & 21,0 \\
\hline 2015 & 35 & 1 (SE) & 2,8 & 146 & 24,0 \\
\hline Total & 624 & 11 & 1,8 & 2.725 & 22,9 \\
\hline
\end{tabular}

Figura 2. Negativas judiciales por comunidades autónomas ( $N$ absoluta), España 2014.

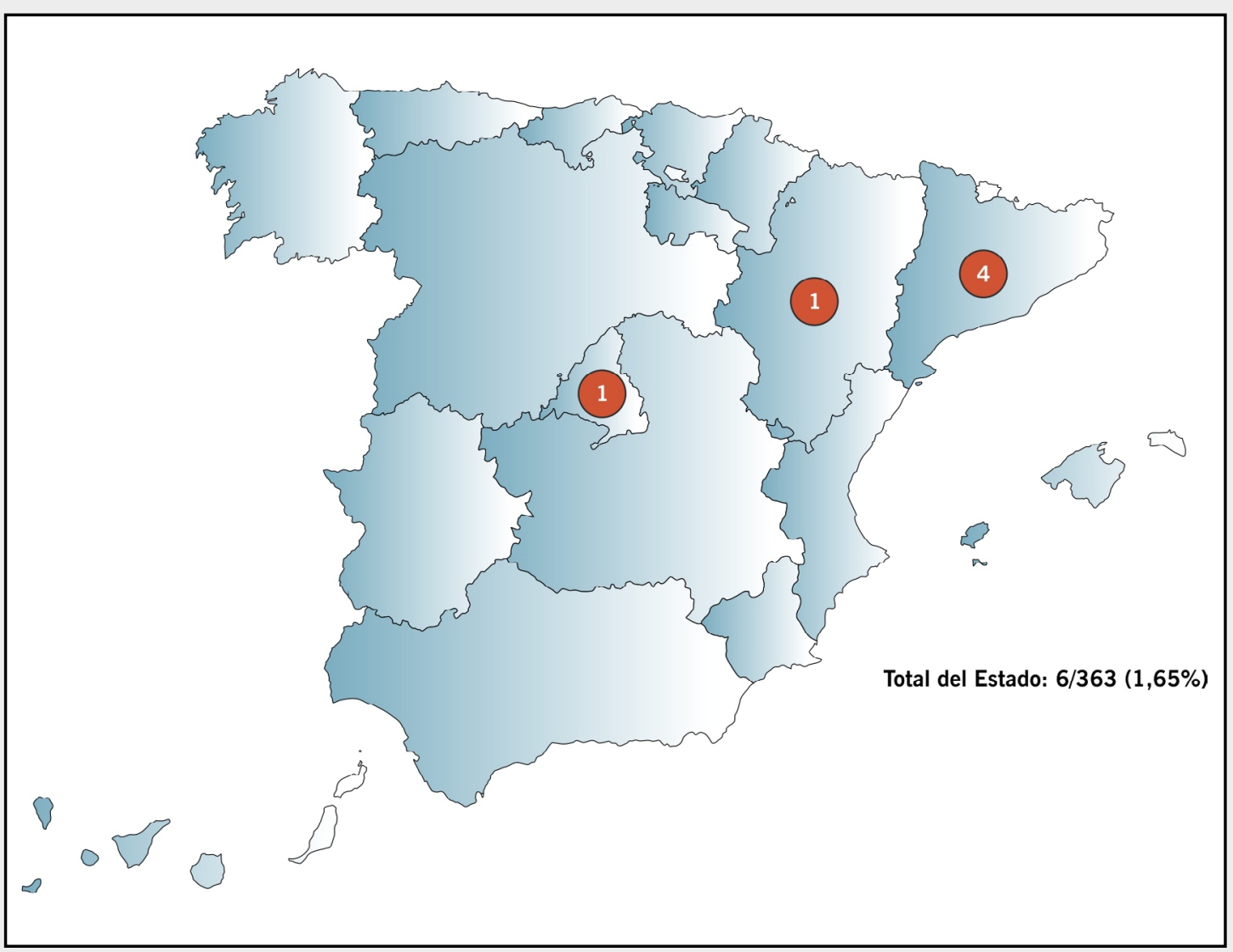



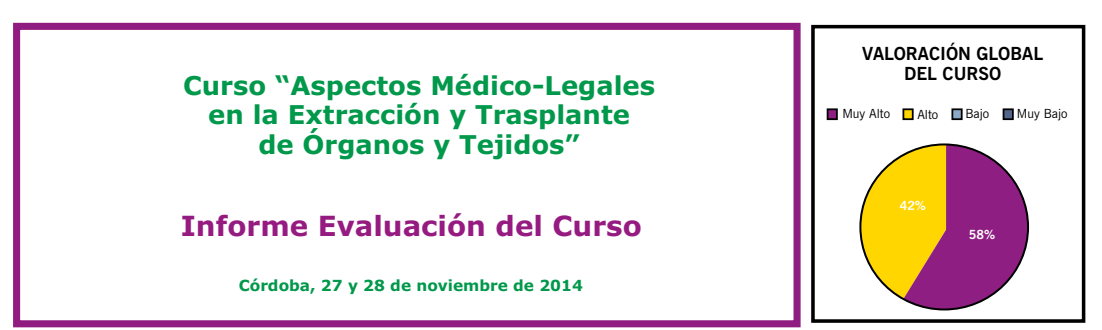

Este curso, se celebró en Córdoba con la asistencia de 67 discentes, 30 hombres y 37 mujeres. Entre los profesionales asistentes a este curso, se encontraban 24 Magistrados y/o Jueces, 26 Médicos Forenses y 17 Coordinadores de Trasplantes, de todas las provincias de Andalucía.
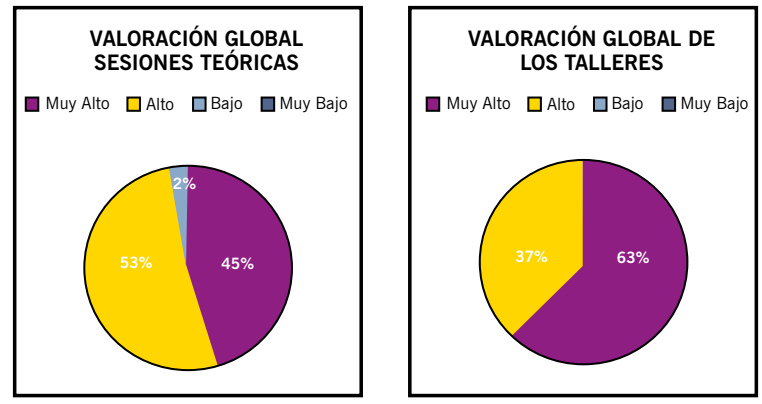

Por último, también se les pedía que puntuaran de 0 a 10 en qué medida recomendarían este curso a otras personas y la puntuación obtenida fue de 9,21 (con una desviación estándar de 0,76).

\section{Curso "Aspectos Médico-Legales en la Extracción y Trasplante de Órganos y Tejidos"}

\section{Informe Evaluación del Curso}

Huelva, 19 de marzo de 2015

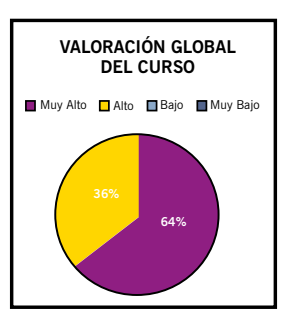

Este curso, se celebró en Huelva con la asistencia de 24 discentes, 10 hombres y 14 mujeres. Entre los profesionales asistentes a este curso, se encontraban 8 Magistrados y/o Jueces, 9 Médicos Forenses y 7 Coordinadores de Trasplantes, 5 de los hospitales de la provincia de Huelva y dos procedentes de Latinoamérica que se encuentran en Andalucía con el proyecto Master Alianza
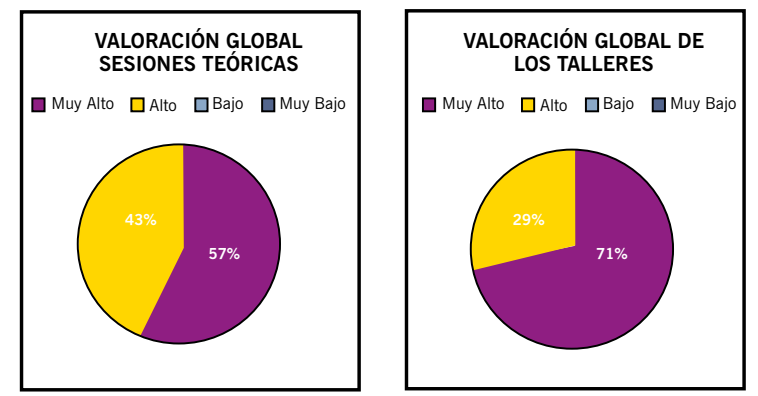

Por último, también se les pedía que puntuaran de 0 a 10 en qué medida recomendarían este curso a otras personas y la puntuación obtenida fue de 9,36 (con una desviación estándar de 0,63).
Figura 3.

Valoración del curso de Córdoba.

\section{Figura 4.}

Valoración del curso de Huelva. 
Figura 5.

Años de vida ganados por una donación.
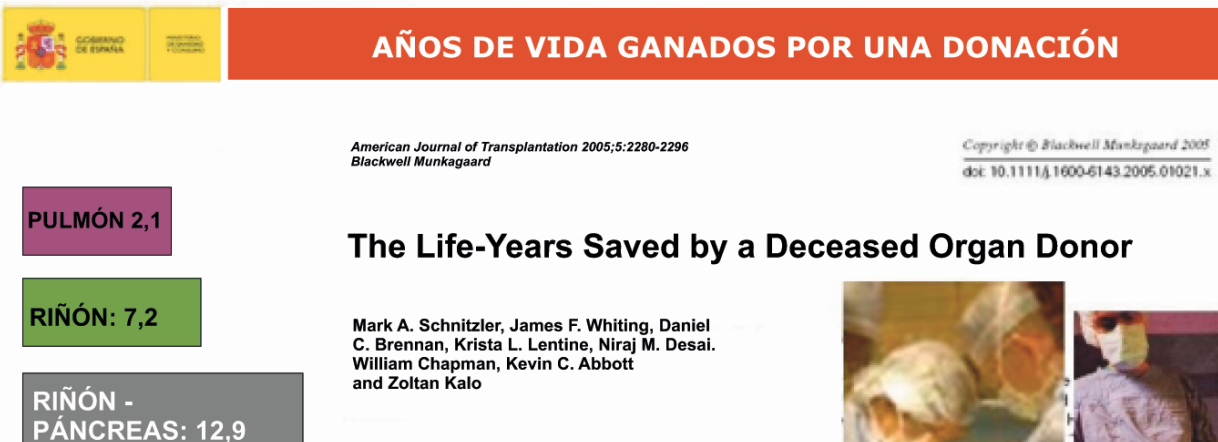

The Life-Years Saved by a Deceased Organ Donor

PÁNCREAS: 12,9

CORAZÓN: 14,5

HÍGADO: 16,1 AÑOS

Mark A. Schnitzler, James F. Whiting, Daniel C. Brennan, Krista L. Lentine, Niraj M. Desai. William Chapman,
and Zoltan Kalo

\section{HIGADO: 16,1 ANOS}

DONANTE PROMEDIO: 30,8 AÑOS

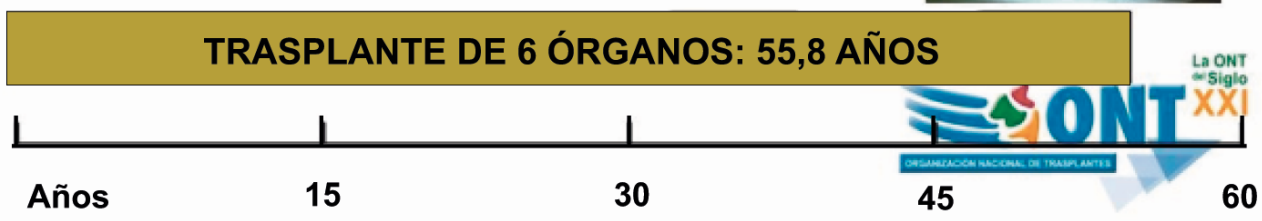

así lo hemos organizado, y ya tuvimos una primera reunión el pasado 19 de marzo en Huelva. Como puede verse en las Figuras 3 y 4, donde se recogen los rasgos principales del informe de evaluación que sistemáticamente realizamos después de cada curso o jornada, el nivel de aceptación es muy elevado. Y no resulta fácil en algunos casos organizar estos eventos, ya que, hoy día, por motivos laborales, es complejo conseguir la disponibilidad de todos los que desean asistir. Y como ya lo sabemos, los próximos cursos los organizaremos durante las tardes de 2 días para que haya más posibilidades de acudir.

Una vez que han conseguido su plaza por oposición y antes de comenzar a trabajar en su primer destino, los jueces disponen de un periodo de formación específica en distintos temas, entre ellos la donacióntrasplante. Desconocemos si los médicos forenses tienen oportunidades similares. En todo caso, estas jornadas sólo pretenden conseguir una puesta en común y ser complementarias con la formación que se haya podido adquirir al respecto por los medios que sean.

En resumen, como Coordinador Autonómico de Trasplantes de Andalucía, quiero destacar el formidable apoyo y colaboración que recibimos de jueces y forenses en el trabajo diario de posibilitar la donación de órganos y tejidos. Es de significar que no hay ningún proceso asistencial que genere más vida o más años de vida ganados que una donación de órganos (Figura 5). En un $23 \%$ de los donantes es preceptivo contar con la autorización judicial para la extracción de órganos y tejidos. $Y$ aunque el porcentaje de aceptación es altísimo, habría que intentar, si fuera posible, llegar al 100\%. O dicho de otra manera, todos los profesionales mencionados en este escrito somos servidores públicos y es legítimo que nos encontremos muy orgullosos de nuestro trabajo. Pero, aunque estemos contentos, no debemos estar satisfechos. Siguen falleciendo todos los años entre un $5 \%$ y un $10 \%$ de pacientes a los que no 
llega a tiempo el órgano que esperan. Lo mismo que aspiramos a conseguir el $0 \%$ de rechazo en la ciudadanía a la donación de órganos, debemos intentar conseguir el $0 \%$ de negativas judiciales. Las personas fallecidas que son potenciales donantes están hoy día muy bien estudiadas desde el punto de vista clínico, y los adelantos científicos contribuyen cada vez más al conocimiento de la causa de la muerte, factor clave para poder autorizar la extracción de órganos y tejidos. Perder un donante implica dejar de salvar unas cuantas vidas humanas. Me consta que ese es el mismo objetivo y deseo de jueces y forenses, a quienes, en nombre de todos los coordinadores de trasplantes y de los pacientes ya trasplantados, les reitero mi gratitud por su impecable profesionalidad y por su contribución desde su trabajo a salvar vidas humanas. 